\title{
Comparative noise performance of a coded aperture spectral imager
}

\author{
Jonathan Piper ${ }^{\mathrm{a}}$, Peter Yuen ${ }^{\mathrm{b}}$, Peter Godfree ${ }^{\mathrm{a}}$, Mengjia Ding ${ }^{\mathrm{b}}$, Umair Soori $^{\mathrm{b}}$, Senthurran Selvagumar ${ }^{\mathrm{b}}$ \\ and David James \\ ${ }^{\mathrm{a}}$ Defence Science and Technology Laboratory, Porton Down, Salisbury, SP4 0JQ, UK; \\ ${ }^{\mathrm{b}}$ Cranfield University, Defence Academy of the United Kingdom, Shrivenham, SN6 8LA, UK
}

\begin{abstract}
Novel types of spectral sensors using coded apertures may offer various advantages over conventional designs, especially the possibility of compressive measurements that could exceed the expected spatial, temporal or spectral resolution of the system. However, the nature of the measurement process imposes certain limitations, especially on the noise performance of the sensor. This paper considers a particular type of coded-aperture spectral imager and uses analytical and numerical modelling to compare its expected noise performance with conventional hyperspectral sensors. It is shown that conventional sensors may have an advantage in conditions where signal levels are high, such as bright light or slow scanning, but that coded-aperture sensors may be advantageous in low-signal conditions.
\end{abstract}

Keywords: Hyperspectral, imaging spectroscopy, coded aperture, Hadamard spectroscopy

\section{INTRODUCTION}

Over the last decade developments such as the "coded aperture snapshot spectral imager" (CASSI) ${ }^{1}$ concept and others $^{2,3}$, have stimulated interest in a category of sensors that combine coded apertures with conventional spectrographs to create a new kind of hyperspectral imager. Such sensors offer the potential to make compressive measurements and therefore exceed the performance that would normally be expected given their characteristics such as frame rate and numbers of spatial resolution elements. Such systems may be particularly useful for reducing size, weight and power requirements and could therefore make it easier to deploy spectral sensors on smaller platforms, including uninhabited vehicles. They may also offer other advantages, such as the facility to configure the measurement process to optimise for a particular characteristic (e.g. spectral, spatial or temporal resolution) using spatial light modulators and embedded software. However, the nature of the measurement process imposes constraints on other aspects of performance, particularly signal-to-noise ratio (SNR). These trade-offs have been shown to have significant impact on the utility of the resulting data for specific remote sensing tasks. ${ }^{4,5}$

This paper examines the circumstances under which a particular sensor of this type may be expected to provide a benefit over conventional systems. The analysis is carried out in terms of SNR. Like the CASSI system, which is designed for compressive sensing, the sensor analysed here collects multiplexed measurements, in the sense that each measurement is a linear combination of many elements of the signal. However, unlike the CASSI system, it is assumed that the sensor considered in this work is operated in a pushbroom mode in order to provide a complete (as opposed to compressive) measurement. An analytical expression for the SNR is derived and used to examine trends in performance, compared with a conventional non-multiplexed pushbroom hyperspectral sensor. Subsequently the SNR is assessed for model parameters intended to be representative of realistic systems. It is shown that conventional sensors usually have an advantage in conditions where signal levels are high, such as bright light or slow scanning, but that the coded-aperture sensor may be advantageous in low-signal conditions. This result is consistent with the conventional understanding of the multiplex advantage: the multiplexed sensors would be expected to be superior where the dominant noise source is independent of the signal level (which is the case in low-light), but multiplexing should be a disadvantage in brighter light where shot noise is more significant. ${ }^{6}$

\section{SNR MODEL}

\subsection{Sensor architecture}

The particular sensor configuration considered in this paper uses a conventional imaging spectrograph with the entrance slit replaced by a 2-dimensional coded aperture (sometimes described as a "single-disperser coded aperture snapshot 
spectral imager") $)^{7}$, as illustrated in Figure 1. Unlike a conventional dispersive hyperspectral imager, such a system produces images at the detector array with significant spatial extent in the direction in which different wavelengths are dispersed. No assumption is made about the type of spectrograph; although it is depicted with a prism, it could also be a grating-based system or a combination of both. This arrangement produces the type of measurement illustrated in Figure 2. It is assumed that the size of a monochromatic image in the plane of the detector array is at least twice that of the detector array in the direction of spectral dispersion (which places a minimum constraint on the size of any field stop in the coded aperture plane). This ensures that each detector pixel could be illuminated by a complete spectrum (assuming the appropriate elements of the aperture code were open).

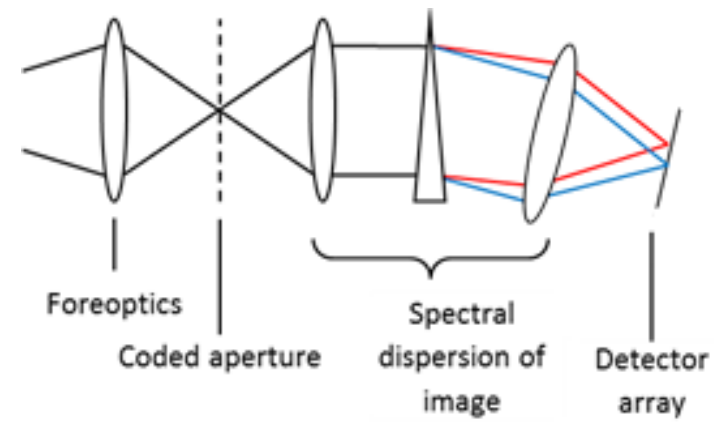

Figure 1. Schematic depiction of the sensor architecture.

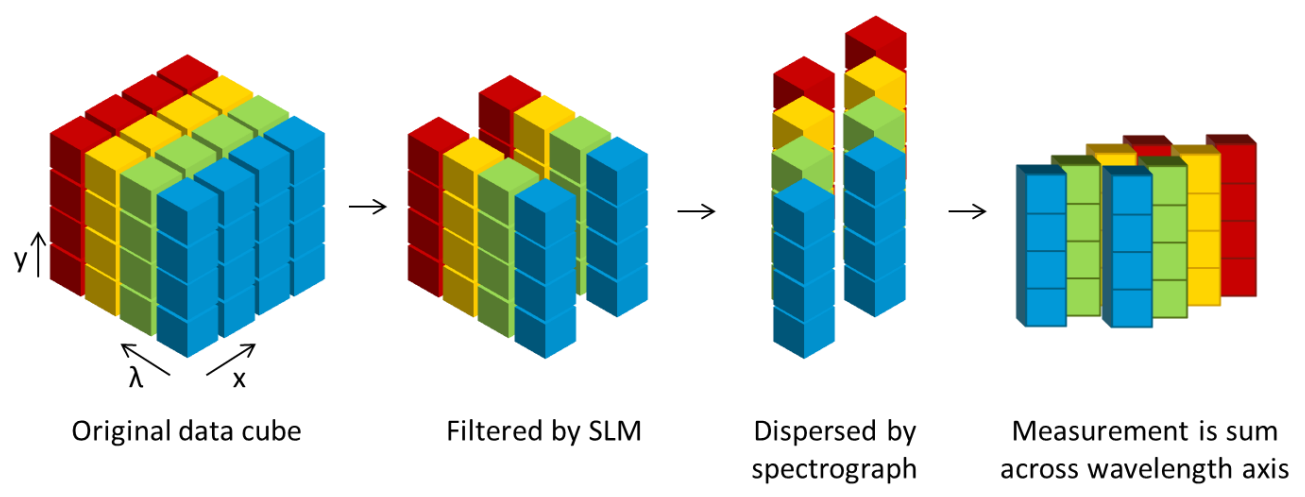

Figure 2. Illustration of how elements of a hyperspectral data cube contribute to measurements from the sensor depicted in Figure 1. A data cube (with two spatial dimensions, $x$ and $y$, and one spectral dimension, $\lambda$ ) is filtered by an aperture code in an un-dispersed image plane, removing light of all wavelengths at particular spatial locations. The light is dispersed by the spectrograph and re-imaged onto a detector, which responds to light of all wavelengths so effectively produces the sum of intensities of voxels across the wavelength dimension.

A code based on a binary Hadamard matrix is used for the coded aperture, the pattern of which is fixed. Such codes have been shown to produce optimal SNR under certain assumptions about the noise model for the system. ${ }^{6}$ The extent to which these conditions are likely to be fulfilled is considered in the next section. The aperture modulates amplitude (not phase). Its elements are assumed to be either entirely opaque or entirely transparent and are imaged to a size equal to one detector element in the plane of the detector array. The same code is applied to every column of the aperture; that is, the pattern does not vary in the direction perpendicular to that in which light is dispersed by the spectrograph. The effects of diffraction from small apertures are not considered. A binary Hadamard matrix is produced from the conventional pattern by replacing $1 \mathrm{~s}$ with $0 \mathrm{~s}$ and $-1 \mathrm{~s}$ with $1 \mathrm{~s}$, and removing the row and column that contain only $1 \mathrm{~s}$ as shown in Figure 3. The Hadamard matrix must be chosen such that, if its columns are ordered appropriately, the columns of the resulting binary matrix are cyclic shifts of the adjacent columns (not all Hadamard matrices have this property). The code is formed from a binary matrix of size equal to the number of elements in the detector array in the direction of spectral dispersion; this is also the number of wavebands that will be obtained from the sensor (this places a small constraint on the choice of number of wavebands, since Hadamard matrices do not exist for all sizes). If $N$ is the 
number of wavebands, the code contains $2 \mathrm{~N}-1$ elements and is created from the matrix row by repeating elements in the same sequence up to that length.

\begin{tabular}{cccccccc|}
1 & 1 & 1 & 1 & 1 & 1 & 1 & 1 \\
1 & -1 & -1 & -1 & 1 & -1 & 1 & 1 \\
\hline 1 & -1 & -1 & 1 & -1 & 1 & 1 & -1 \\
1 & -1 & 1 & -1 & 1 & 1 & -1 & -1 \\
1 & 1 & -1 & 1 & 1 & -1 & -1 & -1 \\
1 & -1 & 1 & 1 & -1 & -1 & -1 & 1 \\
1 & 1 & 1 & -1 & -1 & -1 & 1 & -1 \\
1 & 1 & -1 & -1 & -1 & 1 & -1 & 1 \\
\hline
\end{tabular}

\begin{tabular}{|lllllll|l|}
\hline 1 & 1 & 1 & 0 & 1 & 0 & 0 \\
1 & 1 & 0 & 1 & 0 & 0 & 1 \\
1 & 0 & 1 & 0 & 0 & 1 & 1 \\
\hline 0 & 1 & 0 & 0 & 1 & 1 & 1 \\
\hline 1 & 0 & 0 & 1 & 1 & 1 & 0 \\
\hline 0 & 0 & 1 & 1 & 1 & 0 & 1 \\
\hline 0 & 1 & 1 & 1 & 0 & 1 & 0 \\
\hline
\end{tabular}

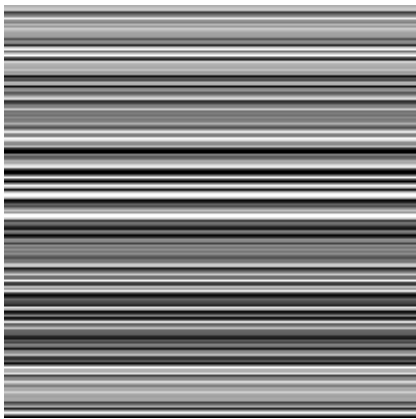

Figure 3. Left - one of the Hadamard matrices of size 8, derived from the quadratic residue construction which is assumed throughout this analysis. ${ }^{6}$ Centre - the corresponding cyclic binary Hadamard matrix. Right - example aperture code for the type of sensor under consideration. Each column follows the pattern of a column (or row) of a cyclic binary Hadamard matrix, repeated cyclically to the appropriate length.

In order to acquire additional information about the signal the field of view of the sensor is scanned in the direction parallel with the spectral dispersion of the image, in the manner of a pushbroom imager. This technique has been demonstrated with the CASSI system using compressive sensing for signal recovery, and shown to improve performance compared with a single snapshot. ${ }^{8}$ For the present analysis it is assumed that $N$ image frames are captured, so that the signal recovery problem is completely determined. It is assumed that the rate of scanning is such that the image advances one pixel for each frame. In a practical system it would be necessary to register consecutive frames in order to aggregate the information they contain. Registration errors would result in degradation of performance, but this has not been considered in the present analysis.

\subsection{SNR model}

Consider a discrete representation of a hyperspectral data cube $\boldsymbol{F}$ of $L$ spatial samples, $M$ lines and $N$ bands. This may be understood to consist of random variables representing the number of photoelectrons collected by the sensor due to each voxel of the data cube, in which case it could be represented as $\boldsymbol{F} \in \mathbb{Z}_{+}^{L \times M \times N}$. However, in the sensor architecture described above there is no multiplexing between elements of the signal from different spatial samples, and the signal recovery method will not make use of information from other spatial samples, so we may restrict our analysis to the $M \times N$ matrix $\boldsymbol{F} \in \mathbb{Z}_{+}^{M \times N}$ without loss of generality.

Within a particular spatial sample, let $Y_{i, j}$ be a random variable representing the number of photoelectrons collected by detector element $j$ in frame $i$. As described above, we assume there are $\mathrm{N}$ pixels per spatial sample, so $j=1 . . N$; we consider $N$ frames, so $i=1 . . N$; and the scan rate is such that $Y_{i, j}=Y_{i-1, j-1}$. Let $h_{k} \in\{0 . .1\}$ denote the transparency of the $k^{\text {th }}$ element of the aperture code. As described above, $k=1 . .2 N-1$. In this case,

$$
Y_{i, j}=\sum_{m=1}^{N} k_{j-m+N} F_{m, i+j-m}
$$

Form the vector $\boldsymbol{y}=\left(\begin{array}{llll}Y_{1, N} & Y_{2, N-1} & \cdots & Y_{N, 1}\end{array}\right)^{\boldsymbol{T}}$. Then,

$$
y=\left(\begin{array}{c}
h_{2 N-1} F_{1, N}+h_{2 N-2} F_{1, N-1}+\cdots h_{N} F_{N, 1} \\
h_{2 N-2} F_{1, N}+h_{2 N-3} F_{1, N-1}+\cdots h_{N-1} F_{N, 1} \\
\vdots \\
h_{N} F_{1, N}+h_{N-1} F_{1, N-1}+\cdots h_{1} F_{N, 1}
\end{array}\right)=\left(\begin{array}{ccc}
h_{2 N-1} & \cdots & \\
\vdots & \ddots & \\
& & \\
h_{N} & & h_{1}
\end{array}\right)\left(\begin{array}{c}
F_{1, N} \\
F_{2, N-1} \\
\vdots \\
F_{N, 1}
\end{array}\right)=H f
$$

With an aperture code constructed as described above, the matrix $H$ takes the form of a cyclic binary Hadamard matrix.

The actual measurements recorded by the detector do not just include $\boldsymbol{y}$; they also include contributions from dark current $(d)$ and the effect of various noise sources that do not depend on the level of the signal $(r)$. We may write the total number of electrons counted in an individual measurement as, 


$$
\boldsymbol{y}_{\text {tot }}=\boldsymbol{y}+d+r
$$

Here $d \sim$ Poisson $(\langle d\rangle), r \sim \operatorname{Normal}\left(0, \sigma^{2}\right)$ and it is understood that addition of a scalar to a vector implies the addition of that scalar to every individual element. The variable $r$ should be understood as a convenient approximation for the effect of a variety of noise sources such as readout noise, quantization noise, etc.

Let $\boldsymbol{\varphi}=H^{-1}\left(\boldsymbol{y}_{\text {tot }}-\langle d\rangle-\langle n\rangle\right)$ represent our estimate of $\boldsymbol{f}$, based on the measurement $\boldsymbol{y}_{\text {tot }}$. It can be seen from (1) and (2) that $\langle\boldsymbol{\varphi}\rangle=\langle\boldsymbol{f}\rangle$; however, $\operatorname{Var}(\boldsymbol{\varphi}) \neq \operatorname{Var}(\boldsymbol{f})$.

Let $H_{i, j}^{-1}$ denote the element of $H^{-1}$ at row $i$ and column $j$; let an asterisk denote every row or column of a matrix (so $H_{j, *}$ is the $j^{\text {th }}$ row of $H$ ); and $\varphi_{n}$ the $n^{\text {th }}$ element of $\varphi$. Then,

$$
\operatorname{Var}\left(\varphi_{n}\right)=\sum_{j=1}^{N}\left(H_{n, j}^{-1}\right)^{2} \operatorname{Var}\left(\boldsymbol{y}_{\text {tot }}-\langle d\rangle-\langle n\rangle\right)=\sum_{j=1}^{N}\left(H_{n, j}^{-1}\right)^{2}\left(H_{j, *}\langle\boldsymbol{f}\rangle+\langle d\rangle+\sigma^{2}\right)
$$

The inverse binary Hadamard Matrix $H^{-1}$ has the property that all of its elements have the same absolute value: $H_{i, j}^{-1}=$ $\pm \frac{2}{N+1} \cdot 6$ This implies that $\left(H_{i, j}^{-1}\right)^{2}=\frac{4}{(N+1)^{2}}$ and therefore (3) can be rearranged as,

$$
\operatorname{Var}\left(\varphi_{n}\right)=\frac{4}{(N+1)^{2}} \sum_{i=1}^{N}\left(N\left(\langle d\rangle+\sigma^{2}\right)+\left\langle f_{i}\right\rangle \sum_{j=1}^{N} H_{j, i}\right)
$$

The sum of any row or column in a binary Hadamard matrix of size $N$ is $(N+1) / 2$ (i.e. $\sum_{j=1}^{N} H_{j, i}=\frac{N+1}{2}$ ). Therefore,

$$
\operatorname{Var}\left(\varphi_{n}\right)=\frac{2}{N+1} \sum_{i=1}^{N}\left\langle f_{i}\right\rangle+\frac{4 N}{(N+1)^{2}}\left(\langle d\rangle+\sigma^{2}\right)
$$

This allows us to compute the SNR (i.e. $\left.\left\langle\varphi_{m}\right\rangle / \sqrt{\operatorname{Var}\left(\varphi_{m}\right)}\right)$. In (4) $n$ denotes the waveband of the estimated signal, at least if we assume that we are observing a spatially homogenous target. As the right-hand side of (4) does not depend on $n$, it can be seen that the noise is independent of the wavelength, even when the signal level is wavelength-dependent. This is unlike a typical dispersive sensor. It is also worth noting that, since $\operatorname{Var}\left(\varphi_{n}\right)$ depends more-or-less linearly on the signal level (in the form of $\sum_{i=1}^{N}\left\langle f_{i}\right\rangle$ ), the SNR does not increase with increasing signal as with a conventional sensor (where the SNR depends on the square-root of signal level).

The expression corresponding to (4) for a conventional dispersive pushbroom HSI is $\operatorname{Var}\left(\varphi_{n}^{c}\right)=f_{n}+\langle d\rangle+\sigma^{2}$. However, in order to compare the two results, it is necessary to consider how signal levels would differ between the two in practical situations. Detector elements have a finite well depth, so it may be necessary to use different integration times for each system to avoid saturation. We can relate the signal for each case (in terms of number of photoelectrons) to a rate which is common for both cases: $\boldsymbol{f}^{c}=\dot{\boldsymbol{f}} \Delta t^{c}$ and $\boldsymbol{f}^{m}=\dot{\boldsymbol{f}} \Delta t^{m}$ where the superscript $c$ denotes the conventional sensor, superscript $m$ the multiplexed sensor and $\Delta t$ is the integration time. Because the coded aperture sensor effectively has $\frac{(N+1)}{2}$ times as many slits as the conventional sensor, we may assume as a first approximation that the light levels at the detector will be greater by this factor. Thus when light is abundant we may assume that $\Delta t^{c}=$ $\frac{N+1}{2} \Delta t^{m}$. However, in other situations there may be a constraint on integration time, such as a required frame rate, that means detectors will not be saturated for either sensor. In this case, the integration times will be equal, and the dominant noise source may be the signal-independent term. Using the simplifying assumptions that $\sum_{i=1}^{N}\left\langle f_{i}^{m}\right\rangle=N\left\langle f_{n}^{m}\right\rangle$ and $\langle d\rangle=0$ it can be seen that,

$$
\frac{S N R^{m}}{S N R^{c}}=\frac{1}{\sqrt{N}} \text { if } \Delta t^{c}=\frac{N+1}{2} \Delta t^{m} \text { but } \frac{S N R^{m}}{S N R^{c}}=\frac{N+1}{2 \sqrt{N}} \text { if } \Delta t^{c}=\Delta t^{m} \text { and } \frac{2}{N+1} \sigma^{2} \gg\left\langle f_{n}^{m}\right\rangle \forall n
$$

In other words, the conventional sensor is better than the multiplexed version by a factor of $\sqrt{N}$ when light is abundant, but the multiplexed sensor is better by the same factor when light is scarce. This is consistent with the expectation that where the noise level is approximately independent of the signal multiplexing gives an advantage, but where shot noise is the dominant source of noise it is disadvantageous. ${ }^{6}$ 
Estimating the integration time of the coded aperture sensor using the approximation $\sum_{i=1}^{N}\left\langle f_{i}\right\rangle=N f_{n}$ will be reasonably inaccurate for many, if not all wavebands (i.e. values of $n$ ). Therefore, in the following section we make use of the observation that the total number of non-zero elements in any row of the sensing matrix is approximately $\frac{(N+1)}{2}$, so the maximum value of any individual element of $\boldsymbol{y}$ is approximately the sum of all elements of $\boldsymbol{f}$ greater than the median of $\boldsymbol{f}$.

Finally, it is also worth considering that, where the system is not limited by a constraint on integration time, so that light is abundant, it may be appropriate to average the results of multiple exposures in order to improve SNR.

\section{ANALYSIS}

In this section the results derived previously are used to estimate the SNR of hypothetical sensors employing realistic components. The particular scenario envisaged is a pushbroom system used for remote sensing from an aircraft. Three sensor configurations are considered: separate visible-near infrared (NIR) and shortwave infrared (SWIR) sensors using components representative of systems marketed for commercial use; and a combined visible to SWIR sensor using a single FPA and dual-angle blazed grating, more representative of systems available to government and research users (such as AVIRIS-NG or MaRS).

Because SNR depends on the level of the input signal, a reference radiance curve must be used. It is therefore assumed that the sensor is observing a spatially-uniform lambertian surface with a constant (w.r.t. wavelength) spectral reflectance, illuminated by sunlight. The solar irradiance spectrum used was the "direct normal spectral irradiance" specified in ASTM G-173-03. ${ }^{9}$ Absorption and scattering in the atmosphere between the surface and the sensor are neglected.

Some numerical parameters of the modelled sensors are shown in Table 1.

Table 1. Parameters for the modelled visible-SWIR sensor.

\begin{tabular}{|l|l|l|l|}
\hline Modelled sensor & Vis-SWIR & SWIR & Vis-NIR \\
\hline Number of wavebands & 480 & 288 & 768 \\
\hline Pixel size, $\mu \mathrm{m}$ & 30 & 20 & 8 \\
\hline Well depth, $\mathrm{e}^{-}$ & $5 \times 10^{6}$ & $1.1 \times 10^{6}$ & $9 \times 10^{4}$ \\
\hline Noise floor, $\mathrm{e}^{-}$ & 600 & 150 & 110 \\
\hline Dark current, $\mathrm{e}^{-} / \mathrm{s} /$ pixel & 207 & 100 & 4000 \\
\hline Maximum frame rate, $\mathrm{Hz}$ & 125 & 450 & 170 \\
\hline Row readout time, $\mu \mathrm{s}$ & 16.8 & 7.7 & - \\
\hline f/\# & 2.0 & 2.0 & 2.5 \\
\hline
\end{tabular}

\subsection{Visual representation of SNR}

For both the conventional and multiplexed sensors SNR is a function of both wavelength and integration time. The relationship between the two SNRs is of the form $S N R^{c}=a S N R^{m} / \sqrt{a S N R^{m}+b}$ where $a$ and $b$ are independent of wavelength. For most situations this produces a somewhat linear relationship, becoming less linear as the overall magnitude of the difference becomes larger, as illustrated in Figure 4. In the remainder of this paper SNRs are plotted as a function of integration time for one specific wavelength, chosen to be close to the peak: 650nm for the visible and visible-SWIR sensors and 1700nm for the SWIR sensor. However, the wavelength dependence should not be overlooked.

SNRs are plotted for frame rates ranging from $15 \mathrm{~Hz}$ to a maximum of $340 \mathrm{~Hz}$. Considering a pushbroom sensor with an along-track sampling distance of $1 \mathrm{~m}$, this corresponds to platform speeds from approximately the cruise speed of a small fixed-wing commercial UAV to the speed of sound at sea level.

\subsection{Performance trends}

Figure 5 shows SNR as a function of frame rate for both conventional and multiplexed sensors. The top-left plot demonstrates several features that are common across different sets of input parameters. The conventional sensor's SNR declines continuously with increasing frame rate; at lower frame rates, where the sensor is still photon-noise limited, SNR is approximately proportional to the inverse square root of the frame rate; at higher frame rates, where the noise 
floor dominates, SNR is inversely proportional to frame rate. The multiplex sensor enters the region of inverse square root dependence above approx. $280 \mathrm{~Hz}$ in this case. For most frame rates the integration time for the multiplexed sensor is set so as to avoid saturation, so it is constant with respect to changing frame rate. At frame rates below approx. $60 \mathrm{~Hz}$ the multiplexed sensor shows improvements in SNR as it becomes possible to average progressively more frames.

The remaining three plots in Figure 5 show the effect changing one of the model's parameters but leaving others constant. The changes are a reduced well depth, increased illumination level and a reduced number of wavebands. It can be seen that in each case the performance of the multiplexed sensor relative to the conventional one is reduced as a result of the change.
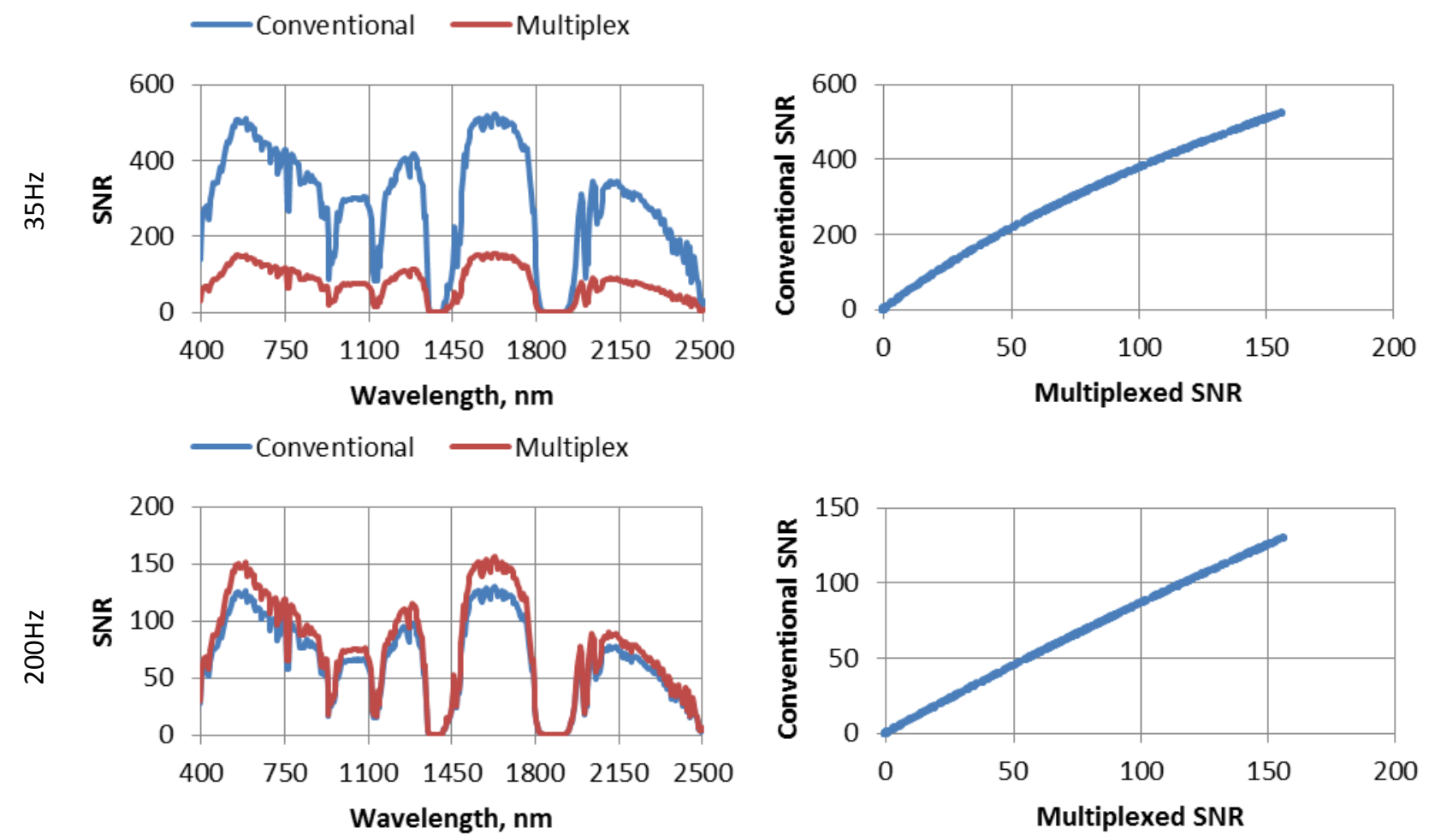

Figure 4. Conventional and multiplexed SNRs plotted as a function of wavelength (left) and compared directly via a scatter plot (right), showing the extent to which comparison at one wavelength is representative of other wavelengths. Plotted for the Teledyne Chroma detector array with 480 bands, a low illumination angle $\left(70^{\circ}\right.$ from zenith), f/2.5 optics and frame rate of $35 \mathrm{~Hz}$ (top) and $200 \mathrm{~Hz}$ (bottom).

\subsection{Visible-SWIR imager}

The parameters used for modelling this sensor type are based on the Teledyne Chroma detector array ${ }^{10}$ and a dual-angle blaze grating such as that described by Mouroulis et al. ${ }^{11}$ The efficiency is adjusted to account for broad-band losses due to a telescope, assumed to be a 3-mirror anastigmat.

Figure 6 shows comparative SNR for two assumed levels of target reflectance, 50\% and 10\%, both assuming an illumination angle of $60^{\circ}$ from zenith. It can be seen that the target reflectance hardly affects the performance of the multiplexed sensor at all, so although the conventional system has a clear advantage at high reflectance, at low reflectance and high required frame rate the multiplexed sensor is superior. Although in this case the target reflectance has been reduced, any effect that reduces the perceived radiance at the sensor, such as increased angle of illumination, would improve the relative performance of the multiplexed system. 


\subsection{SWIR imager}

The parameters used for the SWIR imager are based on AIM's $384 \times 288$ pixel CMT detector array. ${ }^{12}$ Dark current is estimated with the aid of Tennant's Rule $07,{ }^{13}$ assuming an operating temperature of $150 \mathrm{~K}$ (allowing for the rate to exceed that predicted by the rule, which is a lower limit, to a similar extent to the Teledyne detector). The grating efficiency is approximated according to equation (4) from Mouroulis et al ${ }^{14}$ with a peak efficiency of 0.9 and a blaze wavelength of $1700 \mathrm{~nm}$ (operating in the $1^{\text {st }}$ order); this blaze angle produces reasonable efficiency at the longwavelength end of the spectrum, at the cost of efficiency dropping below $15 \%$ at $1000 \mathrm{~nm}$. Transmission of the objective lens is estimated based on a refractive SWIR lens from Optec S.p.A.

It can be seen from Figure 7 that in this case the conventional sensor outperforms the multiplexed version for all frame rates and both reflectance levels. However, it is interesting to note that at lower frame rates the multiplexed SNR is actually better than for the Teledyne detector which has much higher well depth. This is due to the higher maximum frame rate which allows averaging of more frames. This suggests that detector arrays with faster read out times may make multiplexed sensors more feasible. However, where there is a trade-off between number of wavebands (recall that more bands improves the relative performance of multiplexing) and frame rate, the effect of changing the number of bands is not intuitively obvious.
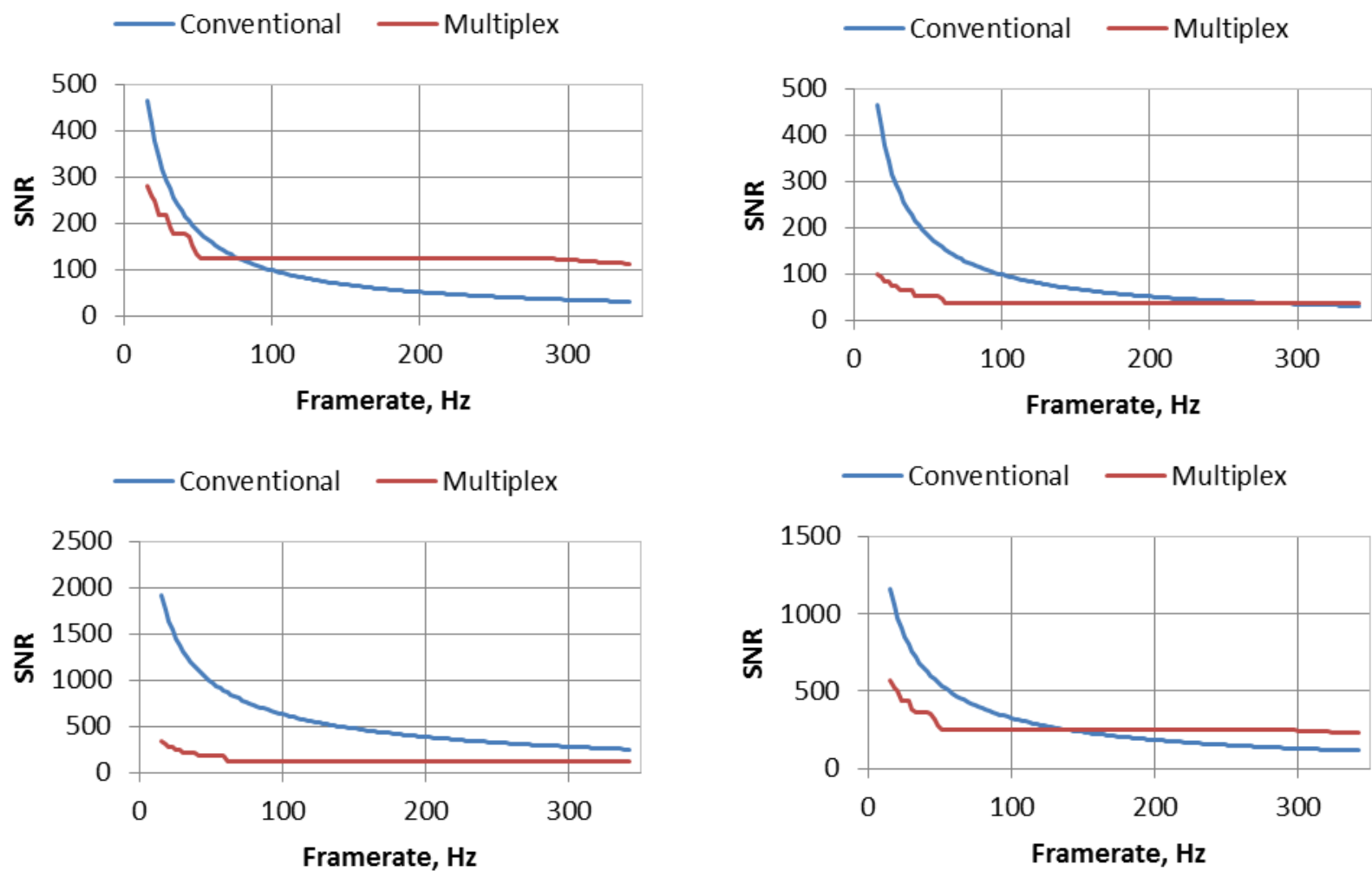

Figure 5. SNR as a function of frame rate for conventional and multiplexed sensors. Top-left shows results for visible-SWIR configuration with $10 \%$ target reflectance and illumination $60^{\circ}$ from zenith. Other plots show the effect of changing one or two parameters but keeping all others constant. Top-right: well depth reduced to $700,000 \mathrm{e}^{-}$(from 5.000,000 $\mathrm{e}^{-}$); bottom-left: $50 \%$ target reflectance and $0^{\circ}$ illumination angle; bottom-right: 120 bands, reduced from 480 (but frame read time still fixed at $8.064 \mathrm{~ms}$ ). Plots are continued past the maximum frame-rate for the sensor in order to elucidate trends more clearly. Note the changes in scale of the SNR axis. 


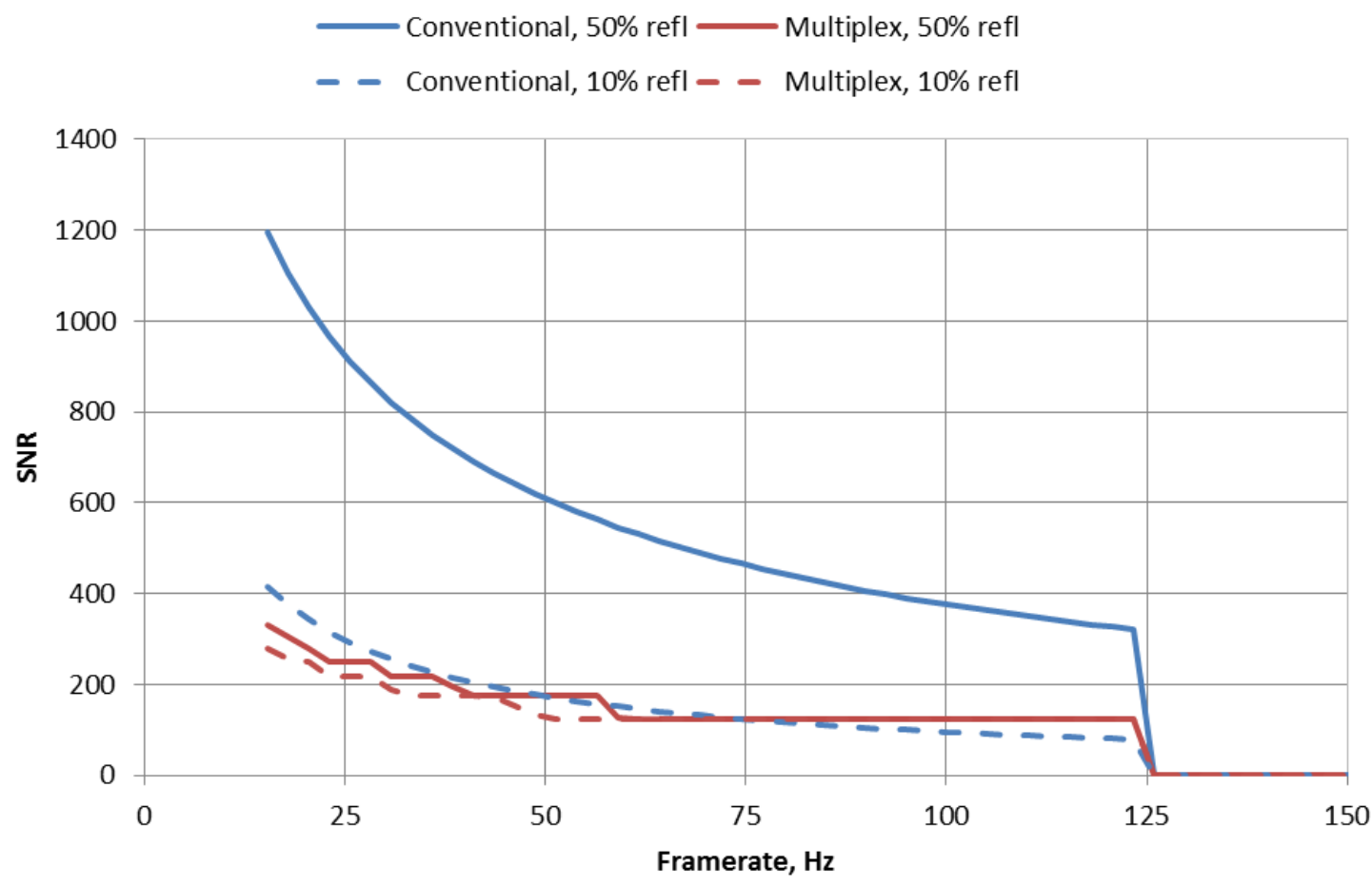

Figure 6. Comparative SNRs for the visible-SWIR sensor.

- Conventional, $50 \%$ refl —Multiplex, 50\% refl

- Convetional, $10 \%$ refl - - Multiplex, $10 \%$ refl

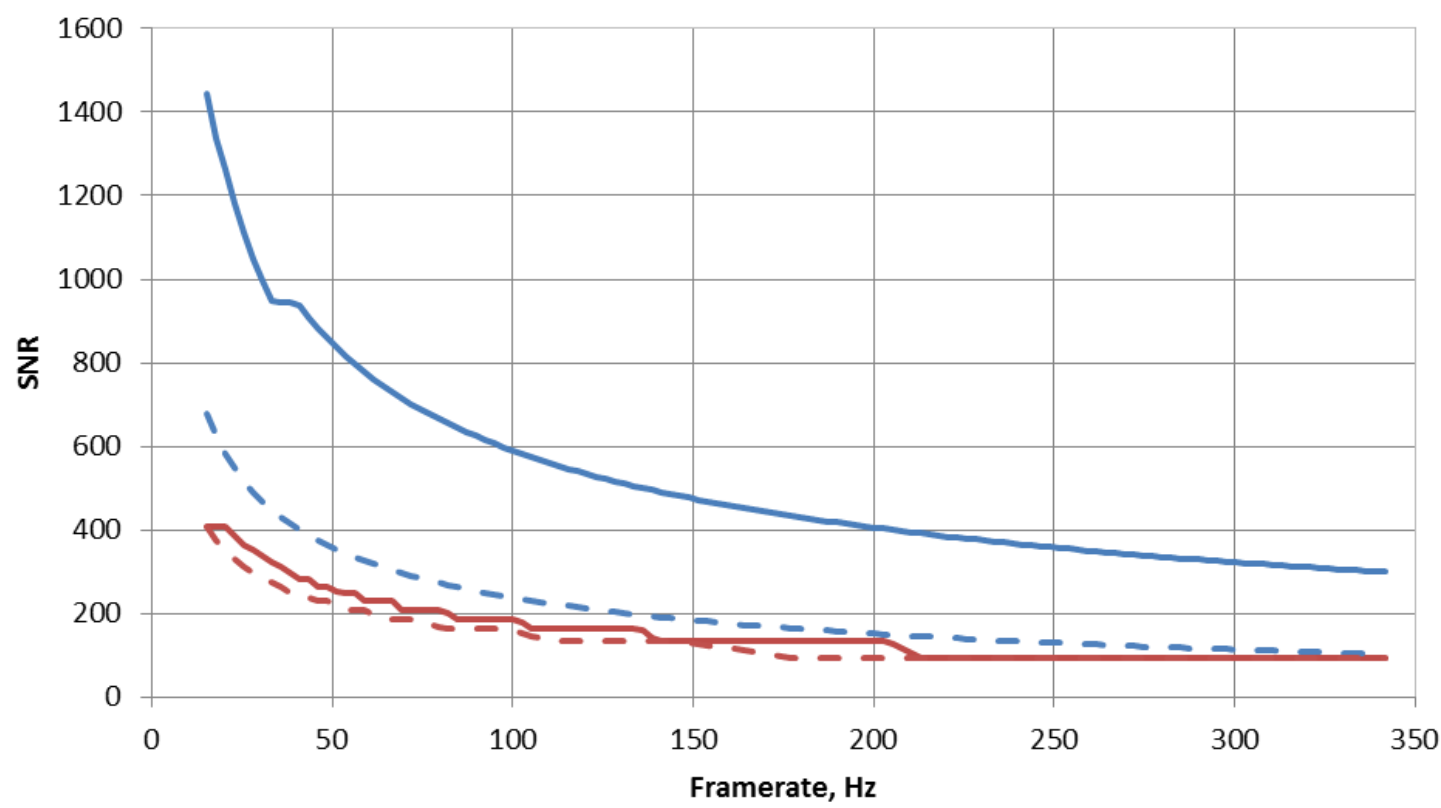

Figure 7. Comparative SNRs for the SWIR sensor. Illumination angle is $60^{\circ}$ from zenith. 


\subsection{Visible-NIR imager}

The choice of a representative detector array for the visible-band imager is more difficult due to the wide variety of options available. The Photonfocus MV1-D1312IE-240-CL has been selected as typical for a mid-range hyperspectral system that is capable but does not use more costly sCMOS detectors. Grating efficiency is approximated in the same way as for the SWIR imager, but with a blaze wavelength of $600 \mathrm{~nm}$. For simplicity, a wavelength-independent $98 \%$ transmission is assumed for the objective lens.

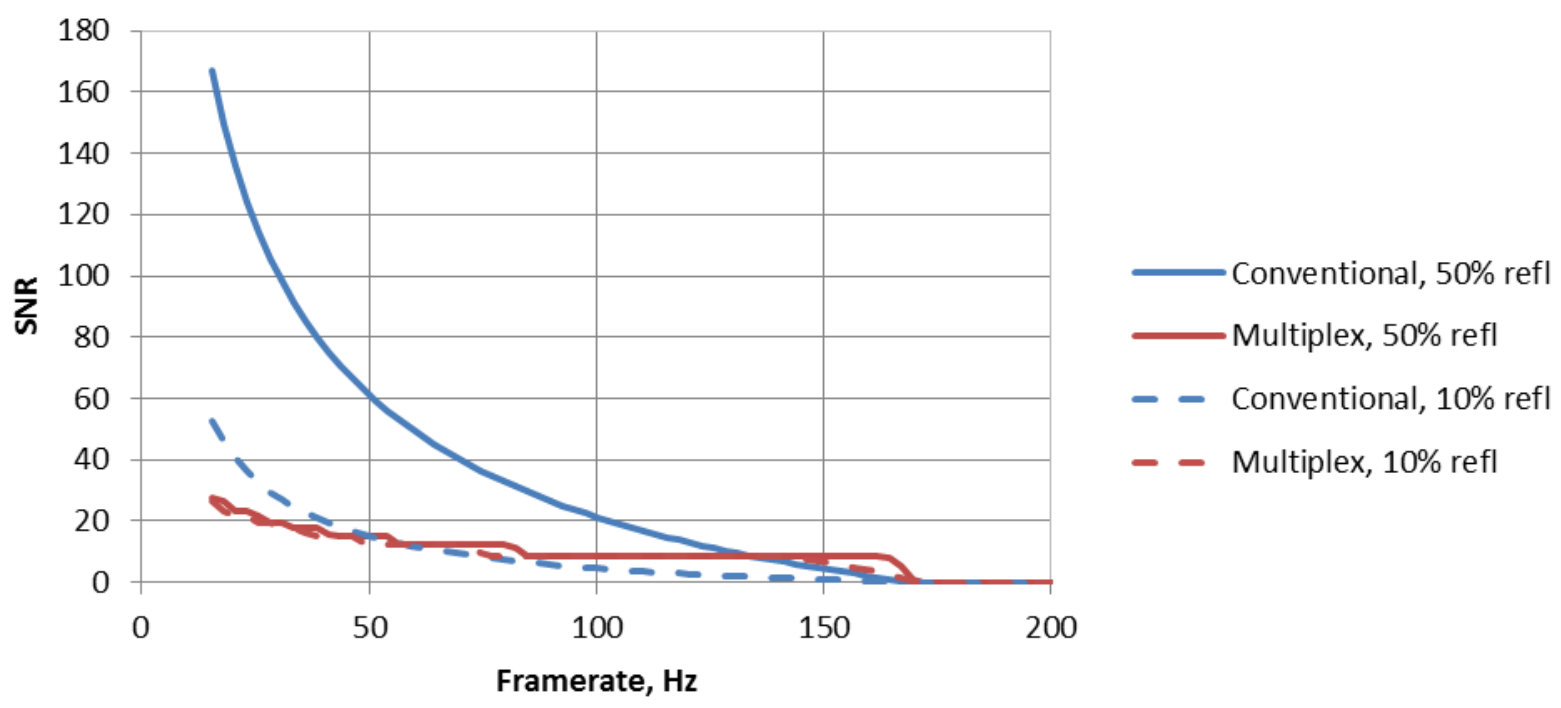

Figure 8. Comparative SNRs for the VNIR sensor. Illumination angle is $60^{\circ}$ from zenith.

Figure 8 shows the multiplexed sensor exceeding the performance of the conventional one for a large proportion of the frame rate range (all frame rates above $\sim 60 \mathrm{~Hz}$ ), for the low target reflectance case. However, the conventional system still dominates for high target reflectance. It should be noted that for this system all the SNRs are poor, and that therefore a system based on these components may not be well-suited to remote sensing applications.

\subsection{SNR requirements for remote sensing tasks}

Work within Dstl suggests that a peak SNR of 75 is sufficient to detect the majority of full-pixel, spectrally-distinctive targets in combined visible-SWIR imagery. For sub-pixel targets of $12 \%$ fill-factor a significant proportion of targets will not be detected for peak SNRs of 250 and below. For sub-pixel targets of $6 \%$ fill-factor a significant proportion of targets will be missed at peak SNRs of 400 and below. These results are based on a typical workflow using QUAC ${ }^{15}$ for atmospheric compensation and $\mathrm{ACE}^{16}$ for target detection, with a fixed detection threshold intended to exclude false alarms due to sensor noise.

\subsection{Comparison with compressive measurement schemes}

It is interesting to consider to what extent the above results could be generalised to sensors using the same multiplexing architecture but a compressive sensing approach to recovering the signal. The quality of recovery in compressive sensing generally improves with more measurements, and this has been shown experimentally with hyperspectral data. ${ }^{8}$ However, the addition of prior information, in the form of expectations about the sparsity of a signal, might be expected to improve the quality of results compared with those presented here, given the same quantity of measurements as are used here. Compressive sensing is often assumed to be a way to trade data fidelity for frame rate (for example to produce spectral video), but it is possible that it may provide higher fidelity measurements under certain conditions.

\section{SUMMARY}

This paper has presented detailed calculations of the noise performance of multiplexing imaging spectrometers using coded apertures, based on the capabilities of real components and including many of the considerations that affect the performance of these systems. Comparison with similar estimates for conventional dispersive sensors shows that the 
coded-aperture systems provide an advantage in low light conditions, or where there are constraints that require high frame rate. In a remote sensing context, such constraints are related to the speed of the platform, and may be derived from a requirement to survey a minimum area in a specified length of time.

However, it is important to note that under a wide range of conditions the multiplexed sensors display poorer SNR than the conventional forms, and even in circumstances where they are better, the SNR they achieve is poor compared with conventional sensors used under optimal conditions. This would limit their usefulness for sub-pixel target detection, although they may still be of use for finding full-pixel targets.

The results are consistent with expectations in that it is understood that the "multiplex advantage" is only actually an advantage when the dominant noise source is independent of the signal level. This is the case in low light or at fast frame rates, where the signal is low and the shot noise is correspondingly low; in brighter light shot noise dominates so the multiplexing effect of the sensor is detrimental not advantageous.

\section{REFERENCES}

[1] Arce, G. R., Brady, D. J., Carin, L., Arguello, H. and Kittle, D. S., "Compressive coded aperture spectral imaging: An introduction," IEEE Signal Processing Magazine 31(1), 105-115 (2014).

[2] Goldstein, N., Vujkovic-Cvijin, P., Fox, M., Gregor, B., Lee, J., Cline, J. and Adler-Golden, S., "DMD-based adaptive spectral imagers for hyperspectral imagery and direct detection of spectral signatures," Proc. SPIE 7210, 721008 (2009).

[3] Graff, D. L. and Love, S. P., "Real-time matched-filter imaging for chemical detection using a DMD-based programmable filter," Proc. SPIE 8618, Emerging Digital Micromirror Device Based Systems and Applications V, 86180F (2013).

[4] Deloye, C. J., Flake, J. C., Kittle, D., Bosch, E. H., Rand, R. S. and Brady, D. J., "Exploitation Performance and Characterization of a Prototype Compressive Sensing Imaging Spectrometer," in [Excursions in Harmonic Analysis, Volume 1: The February Fourier Talks at the Norbert Wiener Center] D. T. Andrews, R. Balan, J. J. Benedetto, W. Czaja, and A. K. Okoudjou, Eds., pp. 151-171, Birkhäuser Boston, Boston (2013).

[5] Busuioceanu, M., Messinger, D. W., Greer, J. B. and Flake, J. C., "Evaluation of the CASSI-DD hyperspectral compressive sensing imaging system," Proc. SPIE 8743, 87431V (2013).

[6] Harwit, M. and Sloane, N. J., [Hadamard transform optics], New York: Academic Press, New York (1979).

[7] Wagadarikar, A., John, R., Willett, R. and Brady, D., "Single disperser design for coded aperture snapshot spectral imaging," Appl. Opt. 47(10), B44-B51 (2008).

[8] Kittle, D., Choi, K., Wagadarikar, A. and Brady, D. J., "Multiframe image estimation for coded aperture snapshot spectral imagers," Appl. Opt. 49(36), 6824-6833 (2010).

[9] ASTM, "G173 Standard Tables for Reference Solar Spectral Irradiances: Direct Normal and Hemispherical on $37^{\circ}$ Tilted Surface," (2012).

[10]Demers, R. T., Bailey, R., Beletic, J. W., Bernd, S., Bhargava, S., Herring, J., Kobrin, P., Lee, D., Pan, J., Petersen, A., Piquette, E., Starr, B., Yamamoto, M. and Zandian, M., "The CHROMA focal plane array: a largeformat, low-noise detector optimized for imaging spectroscopy," Proc. SPIE 8870, 88700J (2013).

[11] Mouroulis, P., Green, R. O., Van Gorp, B., Moore, L. B., Wilson, D. W. and Bender, H. A., "Landsat swath imaging spectrometer design," OPTICE 55(1), 015104-015104 (2016).

[12] Figgemeier, H., Benecke, M., Hofmann, K., Oelmaier, R., Sieck, A., Wendler, J. and Ziegler, J., "SWIR detectors for night vision at AIM," Proc. SPIE 9070, 907008 (2014).

[13] Tennant, W. E., "Rule 07 Revisited: Still a Good Heuristic Predictor of p/n HgCdTe Photodiode Performance?," Journal of Electronic Materials 39(7), 1030-1035 (2010).

[14] Mouroulis, P., Wilson, D. W., Maker, P. D. and Muller, R. E., "Convex grating types for concentric imaging spectrometers," Appl. Opt. 37(31), 7200-7208 (1998).

[15] Bernstein, L. S., Adler-Golden, S. M., Sundberg, R. L., Levine, R. Y., Perkins, T. C., Berk, A., Ratkowski, A. J., Felde, G. and Hoke, M. L., "A new method for atmospheric correction and aerosol optical property retrieval for VIS-SWIR multi-and hyperspectral imaging sensors: QUAC (QUick Atmospheric Correction)," SPECTRAL SCIENCES INC BURLINGTON MA (2005).

[16] Manolakis, D., Marden, D. and Shaw, G. A., "Hyperspectral image processing for automatic target detection applications," Lincoln Laboratory Journal 14(1), 79-116 (2003). 
(C) Crown copyright (2016), Dstl. This material is licensed under the terms of the Open Government Licence except where otherwise stated. To view this licence, visit

http://www.nationalarchives.gov.uk/doc/open-government-licence/version/3 or write to the Information Policy Team, The National Archives, Kew, London TW9 4DU, or email:

psi@nationalarchives.gsi.gov.uk 